\title{
Artificially Intelligent Smart Mirror using Raspberry Pi
}

\author{
Abdullahil Kafi \\ Under-Graduate Student \\ Department of Computer \\ Science and Engineering \\ BRAC University \\ Dhaka, Bangladesh
}

\author{
M. Shaikh Ashikul Alam \\ Under-Graduate Student \\ Department of Computer \\ Science and Engineering \\ BRAC University \\ Dhaka, Bangladesh
}

\author{
Sayeed Bin Hossain \\ Under-Graduate Student \\ Department of Computer \\ Science and Engineering \\ BRAC University \\ Dhaka, Bangladesh
}

\begin{abstract}
In this era of evolving technology, the human life is becoming simpler and time efficient. This paper depicts the design and development of smart mirror which will make our everyday life easier and time efficient. Smart Mirror is a simple mirror which has been enhanced by the help of technology. The aim of the smart mirror is to provide an easy way to information service such as news feeds, weather, clock etc. It also provides some basic AI features like real time interaction with users and so on. The Smart Mirror CPU is the Raspberry Pi 3 computer and the framework that retrieves data from the web through the Wi-Fi connectivity. Through facial recognition and speech recognition model, Smart Mirror can identify the user.
\end{abstract}

\section{Keywords}

Artificial intelligence, speech recognition, facial recognition.

\section{INTRODUCTION}

\subsection{Rationale}

Modern science and technology is now more concerned about designing and developing things that can bring comfort to human life and make it easier. The internet transforms peoples' lives by connecting them more easily to information and other peoples in the virtual world. People are now relying more on devices, like smartphone, that can give them access to lot of information with minimum effort. In this paper, this study was proposed to design of a device called "Smart Mirror" which aimed to provide people with an easy way to access information with minimum effort. It will run on the interface of a mirror and provide productive information such as weather forecast, clock, news feed and so on. The mirror will have artificial intelligence and thus will be able to interact with people in real time. It will be intelligent enough to recognize face and voice of a person and thus will be able to identify users. People will be able to retrieve data from internet by interacting with the mirror directly. The mirror will solve the problems that many people experience every day, getting information without distraction. Before going to bed, the user may want to know whether it will be rain in the next morning so that they can plan their commute. Thus, for every little chore like this, mirror will be able to provide solution and make the life of people comfortable by connecting to huge information.

The rest of the paper is organized as follows. In Section 1.2, background story and related projects have been presented. In Section 2, the overviews of key hardware and software components of the smart mirror have been illustrated. In Section 3, the design and architecture of the system have been explained. Moreover, in section 4, the results of the project have been discussed. In Section 6, conclusion of the paper with future work has been presented.

\subsection{Background of Study}

As the technology and application of digital systems is getting popular, the works on this fields are increasing. Now-a-days the world is getting more into artificial intelligence. The concept is integrating artificial intelligence into people's life [1]. In recent days, the applications of artificial intelligence and its usage in the industry is highly appreciated. The proposed artificially intelligent smart mirror is also a prototype which is from the inspiration of the following fields. However, there were some relevant works on the following field.

The Smart Mirror is a special type of mirror application based on face recognition which provides data feed of various websites and services. Raspberry pi along with webcam and microphone is used in this mirror service. The webcam takes pictures of the person standing in front of the mirror and then sends the image to the pi where the OpenCV application performs a visual analysis and detects the person [2].

Mirror2.0 has the feature of providing news and weather reports as well as providing music and video playbacks. This was done with a MacOS and the music files were fetched from the hard drive of the Mac [3].

The Reveal Project, created in the New York Times research and development, consists of an LCD Display covered by a mirror glass. The device used Microsoft Kinect for real time tracking of user's movements. This module also visualizes different information on its surface, such as calendar, mail, news, mail notification. In addition, it also responds to vocal commands. However, one of the excellent feature of this project was the addition of a medicine box scanner, which allowed the user to buy medicines recognizing their prescriptions [4,5].

Medical Mirror combines computer vision and signal processing technique for measuring the heart rate from the optical signal reflected of the face. High power image processing software components as well as processor was used in this mirror. The prototype consists of LCD display with built-in camera and a two-way mirror fitted onto the frame. The smart mirror recognizes the presence of a user when he or she stands in front of it and, after about 15 seconds, it displays the heart rate below the user's reflected image [6,7].

Voice interfaces are becoming more ubiquitous and are now the primary input method for many devices [8]. The use of the voice and making the AI understanding, the command is a work that the researchers are doing for a long time. The google speech recognition library is very handful in this prospect. Kasmi and Esteves [9] considered the problem of coverting audio commands. The work in [10] proposes a Magical Mirror as an interface to provide basic services. The intended services to offer are interactive TV services. 
From inspiration of the works described above, the proposed work is little different. A working system has been developed which will be an artificial intelligence based service with facial recognition and voice command. The mirror will also have the weather, calendar, clock and news services. However, the tricky part that we introduced is the integration of the AI in a raspberry pi which is a very smart and off-theshelf technology.

\section{PROPOSED SMART MIRROR}

This study was conducted in the Department of Computer Science and Engineering, BRAC university, Dhaka, Bangladesh during the year 2017. The requirements and specifications of the Smart Mirror took inspiration from people's every day devices that they use including PCs, tablets and smartphones. The integrated similar features from each to give the user what they would expect out of a modern 'smart device'. Some basic features like clock, calendar, news feed, etc. which are essential part of the everyday life are included in the smart mirror so that people can now have access to these features more easily than ever. Figure 1 is a schematic view of the smart mirror concept that has been proposed in this study. There are a lot of previous projects related to smart mirror but only few with AI integrated with it. People will be able to interact the mirror through AI in real time and access information available on internet such as accessing maps, weather, news feeds, etc.

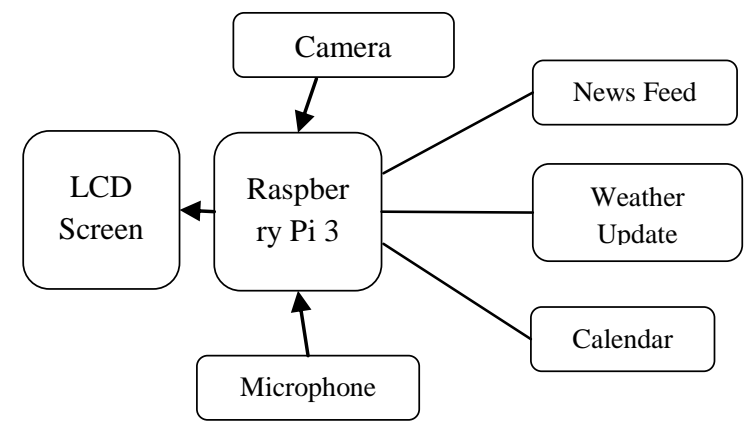

Fig 1: Schematic view of smart mirror

For CPU, Raspberry Pi 3B mini computer was used and all software components were installed into the operating system. The CPU will take the video and audio information from the camera and microphone respectively and through facial recognition and voice recognition model it will identify users. Once the smart mirror senses the presence of any person, it will 'wake up' and display basic features like clock, calendar, weather update, etc. Smart mirror will also have some basic AI features integrated to it. Users will be able to interact with the mirror in real time and search for information on internet, ask questions, perform certain tasks like setting alarm, reminder, etc. with the help of smart mirror. All the information is displayed on the LCD screen connected with the raspberry pi. All components reside behind a special mirror known as two way see through mirror which is made of acrylic material.

\subsection{Hardware components overview 2.1.1 Raspberry Pi}

Raspberry Pi is a credit-card sized computer manufactured and designed in the United Kingdom by the Raspberry Pi foundation with the intention of teaching basic computer science to school students and every other person interested in computer hardware programming and DIY-Do-it Yourself projects. The Raspberry Pi has a Broadcom BCM2837 system on a chip (SoC), which includes 4 ARM Cortex-A53 1.2 GHz cores as the processor, VideoCore IV GPU and with 1.0 gigabyte of RAM. It doesn't include a built in hard disk or solid-state drive, but it uses a microSD card for booting and persistent storage. It also includes Bluetooth 4.1 low energy and a $2.4 \mathrm{GHz} 802.11 \mathrm{n} \mathrm{Wi-Fi}$. The Raspberry Pi 3 computer is one of the key components of this project and we have used Model B of the Raspberry Pi 3. Operating system and all other software were installed and stored in a microSD card.

\subsubsection{Webcam}

A simple USB powered webcam was used for facial recognition. It was used as an input device to take video or image as input.

\subsubsection{LCD Screen}

LCD screen was placed behind the mirror which was used to display the desired information to the user.

\subsubsection{Mirror}

Acrylic two way see through mirror which was a special mirror, was used in this project. Unlike the normal household mirror, the two-way mirror was not painted with a color on the back, instead its left untouched. Thus, it was made reflective on one side and transparent on the other side.

\subsubsection{Microphone}

Microphone was connected with the raspberry pi and was used to input audio information to the CPU. Interaction with the mirror was done over microphone. USB microphones had to be used because the Raspberry Pi does not have regular microphone input.

\subsection{Software Components Overview}

\subsubsection{Raspbian $O S$}

Raspbian is a free operating system based on Debian optimized for the Raspberry Pi hardware. Raspbian comes with over 35,000 packages, pre-complied software bundled in a nice format for easy installation on Raspberry Pi computer.

\subsubsection{NodeJS}

NodeJS is an open-source cross-platform JavaScript run-time environment for executing JavaScript code server-side. It comes included with Electron which is used to launch processes to control things that are not available in web API's such as the sensors and microphones for voice recognition.

\subsubsection{Python}

Python is a widely used high-level programming language for general-purpose programming, created by Guido Can Rossum and first released in 1991. It has lots of support and libraries which makes it very popular among raspberry pi community. Most of the codes of this project were written in python. 


\section{WORKING PRINCIPLE}

Figure 2 shows the overall architecture of the smart mirror.
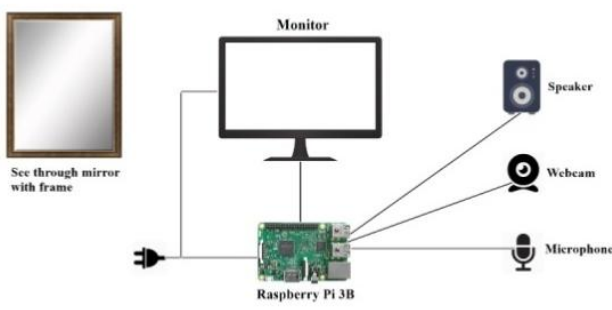

Fig 2. Smart mirror architecture

Figure 2 is a pictorial view of the overall hardware setup. First, a frame for the mirror was built and attached the twoway see through mirror with it. One side of the frame was for the viewing of user and on the other side was assembled all the hardware components. A LCD was attached to display with the frame and connect the LCD display with the raspberry pi via HDMI cable. USB microphone and USB web were then connected with the raspberry pi. Finally, the power source for both the raspberry pi and LCD display was established.

As the raspberry pi has its own operating system, the Raspbian operating system was booted for the project into the raspberry pi. It was updated and upgraded to increase the CPU speed. The default version of the operating system consisted of an older version of the Node which did not consist of NPM. So, the Node v5.1.1 was reinstalled which included the NPM v1.9. Next, pip was installed which was a package installer of python and it helped to install numerous packages. However, python SpeechRecognition 3.8.1 was installed as it was used to convert audio into texts and for processing the video inputs. An OpenCv, a library of programming functions was used which focused on real time computer vision.

Now moving to the coding and designing part, HTML, CSS and JavaScript were used to display the information on the LCD display in such a way that only the information's would appear before the user. And for the AI and other modules, python programming language was used. However, an open source AI library was used to train our AI.

The key features of our design are:

(1) Time and Date: The time of the CPU used (Raspberry Pi) in the mirror was shown

(2) Calendar: The international calendar in the mirror as well as the upcoming holidays was integrated in the system. The help of open source website to fetch the API of the calendar was taken.

(3) News: The news functionality was integrated, which will show the RSS feed of any newspaper of the world.

(4) Artificial Intelligence (AI): Figure 3 shows the working principle of $\mathrm{AI}$.

AI will take voice information as input and through voice recognition, will identify users. Processing audio or speech is more time consuming than processing text. So, the audio input will be converted into text through speech software, here used google speech to perform this task. To make AI understand the text, wit.ai was used which would have intent and entity. Then the AI needed to retrieve relevant information as per user request and this the knowledge phase was called. Once the information is obtained, AI needs to form a complete sentence to express the information, so this is done in the Natural Language Generation state. The AI has a complete sentence, this text has to be converted into speech. Thus, this loop will be continued.

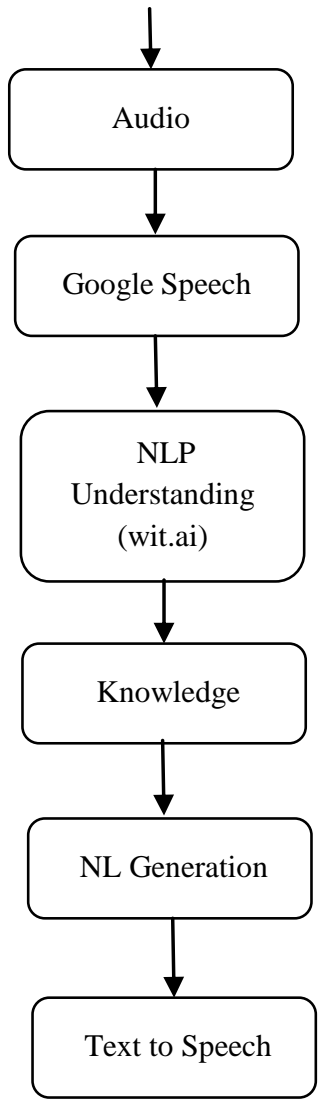

Fig 3: Working principle of AI

\section{PERFORMANCE EVALUATION}

After setting up all the hardware and software components, the model was checked for the outcome. The first launch wasn't successful as the AI wasn't taking any voice command due to the failure of ffmpeg and speech recognizer package. However, the library and package were reinstalled and then launched the model again. This time, it could recognize the voice but the model was responding very slowly.

Next, the problem we faced launching the system was to make the AI understand the different types of entities, as the app was trained under wit.ai. The different entities in every launch was created as the amount of voice command as well as understanding level of AI was increasing. So, some fixed entities were added to enrich knowledge of the AI.

Furthermore, the model was not fast enough as the RAM of the Raspberry Pi was only 1.0 GB and the processor was only 1.2 GHz. However, overclocking the pi processor was tried and came up with a good result. The GPU was decreased to get the maximum amount of speed from the Raspberry Pi.

Table 1. Observation of the performance efficiency in different clock speed

\begin{tabular}{|c|c|c|}
\hline $\begin{array}{c}\text { CPU Speed (arm } \\
\text { speed) }(\mathbf{C C / s})\end{array}$ & $\begin{array}{c}\text { GPU Speed } \\
(\mathbf{C C / s})\end{array}$ & Efficiency (\%) \\
\hline 800 & 126 & 80 \\
\hline 1100 & 126 & 85 \\
\hline 1350 & 16 & 90 \\
\hline
\end{tabular}


Moreover, the data were demonstrated in the table was provided by calculating the CPU usage in different clock speed. In minimal GPU speed and maximum CPU speed the model worked perfectly.

\section{CONCLUSION}

Efforts have been made to build an efficient and intelligent smart mirror that optimizes our time of doing works and increases our daily productivity. The Smart Mirror will play an important role in the field of IoT and home automation. Not only this can function as a normal mirror but can also provide other functionalities like weather forecast, calendar, time, etc. which makes it more desirable. The functionality of the mirror can be expanded by connecting it to other home appliances, mobile devices, etc. Smart Mirror can be a great example of how AI can be integrated into home appliances to make our life easier, efficient and more enjoyable. In future, Smart Mirror can be made smarter by upgrading the AI. There is still a great scope to improve the AI. Soon, normal mirrors will be replaced by smart mirrors if they can be made affordable.

\section{ACKNOWLEDGMENTS}

The authors completed the project not only by the combined efforts of all the authors but also by the help and guidance of their coordinator, Associate Professor Dr. Md. Khalilur Rahman. The authors are really thankful to him for motivating them to finish the project and making this a reality. They are also grateful to Dr. Ayub Hossain for his guidance and help preparing this paper. Moreover, they are thankful to BRAC University and the Department of Computer Science and Engineering for creating such a curriculum that helps Undergraduate Students to accomplish successful events and projects.

\section{REFERENCES}

[1] Piyush, Maheshwari, Maninder, Jeet Kaur, and Sarthak, Anand. 2017. Smart Mirror: A reflective interface to maximize productivity. International Journal of Computer Application. 166(9), 30-35.

[2] Hossain, A.N., Atrey, P.K., Saddik, El. 2007. Smart Mirror for Ambient Home Environment. 3rd IET International Conference on Intelligent Environments, 24-25 Sept. 2007.

[3] Aarts, E., Harwig. H., Schuurmans, H. 2001. Ambient Intelligence, The Invisible Future. McGraw Hill. New York, 235-250.

[4] Bomarius, F., Becker, M., Kleinberger, T. 2006. Embedded Intelligence for Ambient Assisted Living. ERCIM News. 67, 19-20.

[5] N. Carlini, P. Mishra, T. Vaidya, Y. Zhang, M. Sherr, C. Shields, D. Wagner, and W. Zhou. 2016. Hidden voice commands. In USENIX Security, Austin, TX, 2016.

[6] Khanna, V., Vardhan, Y., Nair, D., Pannu P. 2017. Design and Development of a Smart Mirror Using Raspberry Pi. International Journal of Electrical, Electronics and Data Communication 5(1), 63-65.

[7] Poh, M.Z., McDuff, D., Picard, R. 2011. A medical mirror for non-contact health monitoring, ACM SIGGRAPH 2011 Emerging Technologies SIGGRAPH'11. New York.

[8] Raisinghani, M.S., Benoit, A., Ding, J., Gomez, M., Gupta, K., Gusila, V., Power, D.,Schmedding, O. 2004. Ambient intelligence: Changing forms of humancomputer interaction and their social implicatiosn. Jounal of Digital Information.

[9] Kasmi, C., Esteves, J.L. 2015. IEMI threats for information security: Remote command injection on modern smartphones. IEEE Transactions on Electromagnetic Compatibility. 57(6):1752-1755.

[10] Ceccaroni, L., Verdaguer, X. 2004. Magical Mirror: Multimedia, Interactive services in Home Automation, Working Conferenceon Advanced Visual Interfaces (AVI), New York (2004), 10-21 